\title{
Visual acuity and intraocular pressure after surgical management of late in-the-bag dislocation of intraocular lenses. A single-centre prospective study
}

\author{
Ditte Artzen ${ }^{1} \cdot$ Branka Samolov $^{2} \cdot$ Mats Lundström $^{3} \cdot$ Per Montan ${ }^{2}$
}

Received: 23 June 2019 / Revised: 22 August 2019 / Accepted: 17 September 2019 / Published online: 6 November 2019

(c) The Author(s), under exclusive licence to The Royal College of Ophthalmologists 2019

\begin{abstract}
Objectives To describe logarithm of the minimum angle of resolution (logMAR) best corrected visual acuity (BCVA) and intraocular pressure (IOP) after surgical management of late in-the-bag dislocation of the intraocular lens.

Subjects/methods In a single-centre prospective study, 165 consecutive cases having surgical correction for late in-the-bagdislocation of intraocular lenses were analysed. One-year follow-up data of BCVA, IOP and a specially created composite variable designated "IOP issue" which considered IOP $\geq 23 \mathrm{mmHg}$, ongoing pressure reducing treatment and previous pressure reducing surgery were compared with baseline values. Logistic regression was used to investigate factors with a possible influence on the results.

Results The vast majority, $80 \%$ of patients, were operated with an anterior approach with repositioning of the present IOL. Remaining patients were managed with lens exchange via a posterior approach combined with a pars plana vitrectomy (PPV). Baseline BCVA values improved from $0.65 \pm 0.48$ to $0.32 \pm 0.41(p<0.001)$, IOP decreased from $20.12 \pm 8.4$ to $18.44 \pm 5.96 \mathrm{mmHg}(p=0.02)$ whereas the proportion of cases with a pressure issue remained unchanged, $47.3 \%$, at the $1-$ year follow-up. Determinants for having a pressure issue at the 1-year follow-up were present a pressure issue at baseline and IOL repositioning with the anterior approach.

Conclusions Both surgical methods provided similar visual rehabilitation to other techniques described in the literature. However, subjects whose IOL was exchanged in combination with a PPV were at lower risk for an IOP issue at the follow-up.
\end{abstract}

\section{Introduction}

Late in-the-bag dislocation of the intraocular lenses (IOL) causing vision impairment is an important and not entirely uncommon complication after cataract surgery. Its cumulative incidence has been calculated to be $0.5-1.0 \%$ within a 10 -year period after the cataract operation $[1,2]$ and may become an increasingly frequent condition due to a broader accessibility to cataract surgery offered at younger age, as seen for instance in Sweden [3], leading to longer duration of pseudophakia [2].

$\triangle$ Per Montan

per.montan@sll.se

1 Praktikertjänst, Läkarhuset Odenplan, Stockholm, Sweden

2 St Erik Eye Hospital, Stockholm, Sweden

3 Department of Clinical Sciences, Ophthalmology, Faculty of Medicine, Lund University, Lund, Sweden
Pathologic findings of the capsular bag [4, 5] of explanted intraocular lenses and risk factors for the complication like pseudoexfoliations, intraoperative zonular dehiscence, glaucoma and high myopia [2, 6-14] are well described in the literature. However, visual outcomes and changes in intraocular pressure of the corrective surgery have been presented in but a limited number of publications presenting more than 50 cases with at least a 6-month follow-up, and there is only one to date that included more than 100 participants completing the study [7-12]. The aim of this prospective study performed at a tertiary referral centre was to analyse vision and IOP and issues related to IOP at 1 year after the surgical repair and to explore various factors that could influence these main outcome measures.

\section{Subjects and methods}

During a 2-year period, consecutive patients operated for late in-the-bag dislocation of the IOL were invited to 
participate in a study assessing clinical characteristics at presentation and at a 1-year follow-up visit after the correcting surgery. Criteria for the surgical management were a total IOL dislocation, IOL decentration causing visual disturbances and/or a decentration threatening to become total. Patient recruitment began in December 2010 and ended in November 2012 and the final follow-up examination took place in November 2013. Whenever possible, the eye unit performing the original cataract operation sent the relevant notes to provide background data. Study data collection included demographics; ocular history preceding the dislocation; biometry; logarithm of the minimum angle of resolution (logMAR) best corrected visual acuity (BCVA) and intraocular pressure (IOP) at presentation (baseline) and at the 1-year follow up; correcting surgical technique and ensuing events. Visual acuity testing was performed using the Early Treatment Diabetic Retinopathy chart at $4 \mathrm{~m}$, IOP was assessed with a Goldman tonometer.

Only two operation methods were used. The dislocated IOL was mainly addressed by an anterior approach with ab externo suturing to the scleral wall [15]. Two $3 \mathrm{~mm}$ grooves were created roughly $2.5 \mathrm{~mm}$ posterior to the limbus $180^{\circ}$ apart. An uncut 10-0 polypropylene suture double-armed with straight needles (Ethicon, Cincinatti, OH, USA) was employed to anchor the IOL by forming a loop around the capsular bag-IOL-haptic complex. A bent 27 gauge guiding hypodermic needle was introduced perpendicularly through the scleral groove and then redirected upward to penetrate through the capsule from underneath between the optic and the haptic. The tip of the guiding needle was exited through a limbal incison, where it was docked with the first needle with the polypropylene thread, Fig. 1(a-c). The docked needle with the thread was retracted through the groove. The docking with the second polypropylene needle through the limbal incison was achieved with the guide inserted $2 \mathrm{~mm}$ to the side of the first groove entry after having been passed in the plane between the iris and the IOL. Once retracted through the groove, the thread formed a loop which was tightened and tied on the scleral wall, the knot being buried in the groove. This procedure was repeated in the second groove $180^{\circ}$ away. Anterior vitrectomy was used only in a small minority of cases together with this procedure. Conversely, pars plana vitrectomy (PPV) was used with the alternative surgical method involving lens explantation through a $7 \mathrm{~mm}$ long limbal incision. A replacement IOL made of polymethylmetacrylate (CZ70BD, Alcon Laboratories, Fort Worth, TX, USA) with small islets on its haptics was used. The same fixating prolene suture as previously mentioned was inserted through scleral grooves and recovered outside the eye and passed through the islets. The IOL was implanted and anchored to the scleral wall by tightening the prolene thread. The limbal incision was sutured with 10-0 ethilon. Thus, neither iris fixation of posterior chamber IOLs nor anterior chamber implantation techniques was employed.

During the clinical course up to the 1-year visit, general monitoring of the patients included examinations within 1 week and then at 1 month after surgery. Persistent pressure problems were assessed at additional visits if necessary. The post-operative treatment included dexamethasone $\quad 0.1 \%$ eye-drops $\quad$ (Isopto-Maxidex ${ }^{\circledR}$, Alcon Nordic) TID tapered over three to four weeks and pressure-reducing eye drops as needed. Prophylaxis against endophthalmitis consisted of preoperative conjunctival rinsing with chlorhexidine $0.05 \%$ solution (Fresenius Kabi) and in-house compounded intracameral a

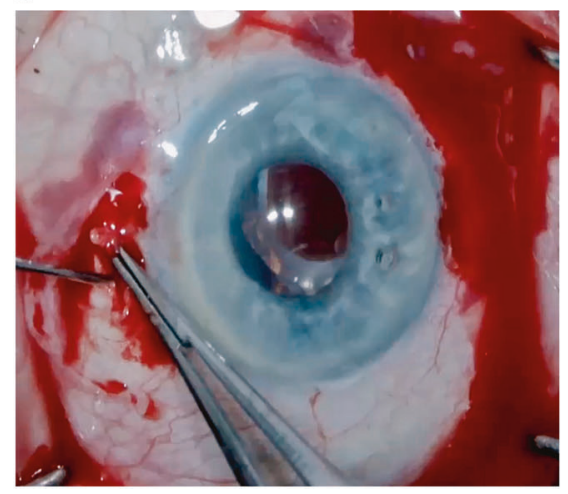

b

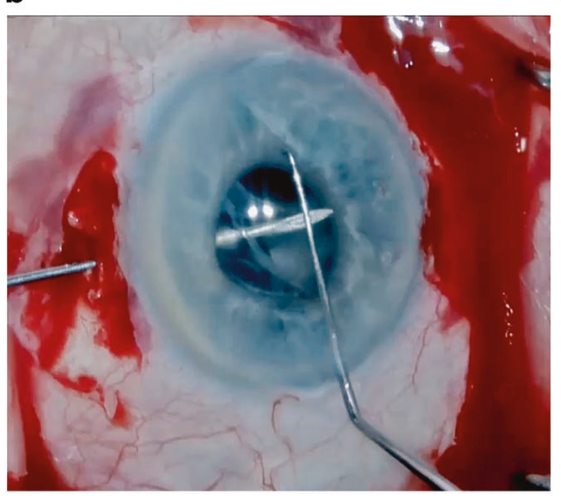

C

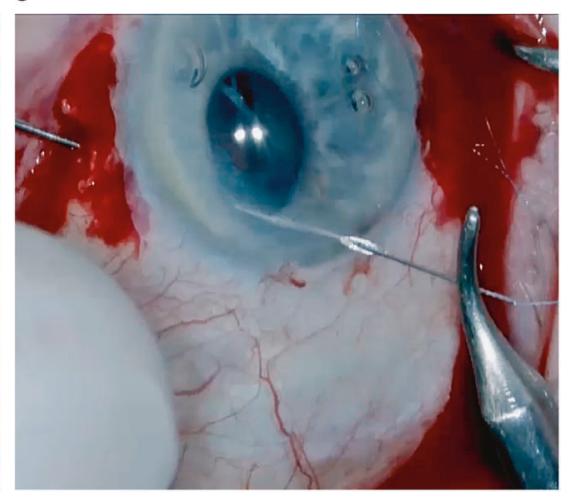

Fig. 1 a-c The initial manoeuvres of an ab externo in situ fixation of an in-the-bag dislocated intraocular lens (IOL). After conjunctival periotomy, scleral grooves are prepared about $2.5 \mathrm{~mm}$ from the limbus. A bent 27 guage guiding needle is introduced through the groove, first perpendicularly to avoid contact with the ciliary body (1a) and then aimed more parallel to the horizontal plane to appear just underneath the IOL optic The needle is used to rotate the IOL to expose the optic-haptic junction. A Sinskey hook is entered into the anterior chamber through a small corneal incision to stabilise the optic and the capsule is penetrated centrally to the haptic with the guding needle (1b). The tip of the guiding needle is externalised through the corneal incision where the docking with the straight needle of an uncut doublearmed $10-0$ prolene suture takes place $(\mathbf{1 c})$ 
$0.2 \mathrm{mg}$ non-preserved moxifloxacin (Vigamox ${ }^{\mathrm{TM}}$, Alcon Nordic) at the end of the procedure.

Primary outcome measures were best corrected logMAR (BCVA), IOP and IOP related issues changes from baseline, i.e. right before the repositioning operation, and at the 1-year follow-up. The latter variable, designated "IOP issue", was composed of any one of (a) previous glaucoma surgery, (b) ongoing pressure reducing medical treatment and (c) an IOP of $23 \mathrm{mmHg}$ or more right before the correcting operation. The same composite variable was created for the 1-year follow up, including any one of (a) glaucoma surgery at the correcting surgery or during the follow-up and (b) and (c) as described above.

Descriptive statistics were given in percentage (categorical variables) or means with standard deviation (continuous variables). The statistical calculations of the main outcome measures were done with appropriate tests for paired data. Risk factors for a BCVA outcome worse than $\log$ MAR 0.3 , for IOP $\geq 23 \mathrm{mmHg}$ and for the presence of a composite IOP issue at the follow-up were tested in single variable calculations. The risk factors tested were patient gender as well as age $\geq 85$ years, BCVA $>0.3 \log$ MAR, presence of pseudoexfoliations, high myopia defined as axial length $>27 \mathrm{~mm}$, total IOL dislocation to the vitreous, IOP $\geq 23 \mathrm{mmHg}$ and presence of IOP issue, all at presentation, in addition to the surgical technique used (anterior or posterior approach, the latter with lens exchange). Any variable associated with a $p$-value of 0.2 or less in these analyses were entered in a subsequent stepwise logistic regression model. Statistical calculations were made with IBM SPSS software version 25 (71S Wacker Dr, Chicago, IL 60606, USA). $P$ values $<0.05$ were considered statistically significant.

Approval of the local ethics committee at the Karolinska Institute was given and informed consent was provided by participating subjects.

\section{Results}

In the 2-year period, 189 eyes in 189 patients were operated for IOL in-the.bag dislocation by seven experienced surgeons. Twenty-four patients failed to report back at the 1-year follow-up leaving 165 cases to be analysed out of whom 57\% were women. For 23 of these cases, there were no data available from the original cataract operation, view Table 1 for ocular history before presentation. Twenty-seven percent of the cataract operation procedures involved at least one of the following manoeuvres: mechanical dilation of small pupils, capsule stabilisation with iris retractors and/or a capsule tension ring (CTR). The latter device was inserted in $18.3 \%$ of these 142 cases. In contrast, the general CTR implantation use in Sweden at this time was reported to be $1.2 \%$ [16]. The mean latency from the cataract operations to surgical repair for dislocation was $9.3 \pm 4.4$ years, but subjects with a CTR implant had a statistically significant shorter latency than those without (Table 2).

At the correcting procedure, mean patient age was $79.2 \pm 8.9$ years. Pseudoexfoliations were present in $80 \%$ and twelve subjects, $7.2 \%$ of the total, had an axial length exceeding $27 \mathrm{~mm}$. These proportions are considerably higher than those found in more recent datasets from the Swedish Cataract Register. Thus, $11 \%$ were found to have pseudoexfoliations in 2013 and $1.9 \%$ had an axial length $>27 \mathrm{~mm}$ in 2015 but these items were not part of the registry in years coinciding with the cataract operation of the study cohort (data on-line through user ID-login).

Mean BCVA was $0.64 \pm 0.48 \log$ MAR (median decimal value 0.3 ), mean IOP was $20.1 \pm 8.4 \mathrm{mmHg}$ while the proportion of participants fulfilling the criteria for a pressure issue was $47.3 \%$ ( $n=78$, Table 2$)$. IOP was not statistically significantly different in eyes with a complete dislocation $(n=25), 18.32 \pm 6.49 \mathrm{mmHg}$, compared with eyes with an IOL in reach from an anterior approach $(n=140), 20.64 \pm$ $8.47 \mathrm{mmHg}, p=0.19$.
Table 1 Ocular history before presentation with late in-the-bag dislocation. Data before and at the cataract extraction available for only 142 patients

\begin{tabular}{ll}
\hline Age & $69.7 \pm 8.4$ years \\
Previous intraocular surgery & $n=9(6.3 \%$, of which 2 trabeculectomies $)$ \\
Glaucoma medication & $n=26(18.3 \%)$ \\
& Cataract extraction manoeuvres $(n=142)$ \\
Pupil manipulation & $n=11(7.7 \%)$ \\
Capsule stabilisation with retractor hooks & $n=17(11.9 \%)$ \\
Capsule tension ring implantation & $n=26(18.3 \%)$ \\
& $\begin{array}{l}\text { Events between the cataract extraction and dislocation }(n= \\
165)\end{array}$ \\
Intraocular surgery save for glaucoma & $n=12(7.3 \%), 6$ of which were vitrectomies \\
Filtering procedure & $n=13(7.9 \%)(11$ trabeculectomies and 2 deep sclerectomies) \\
Blunt trauma & $n=3(1.8 \%)$
\end{tabular}


Table 2 Data at presentation for late in-the-bag dislocation and surgical management, $n=165$

\begin{tabular}{ll}
\hline Gender & Females, $n=94(57 \%)$ \\
& Males, $n=71(43 \%)$ \\
& $79.21 \pm 8.94$ years \\
Age & $9.3 \pm 4.4$ years for all 10.2 \pm 4.2 years \\
Time to dislocation $(n=142)$ & for those without a CTR $(n=117)$ \\
& $5.3 \pm 2.6$ years for CTR implanted $(n$ \\
& $=26)$ \\
& $0.64 \pm 0.47$ logMAR (Median \\
BCVA & decimal equivalent 0.3$)$ \\
& $20.12 \pm 8.4$ \\
IOP & $n=50(30.3 \%)$ \\
Topical treatment for & $n=78(47.3 \%)$ \\
reducing IOP & $n=132(80 \%)$ \\
Pressure issue & \\
Pseudoexfoliations & $n=12(7.3 \%)$ \\
Axial length $>27$ mm & $n=25(15.2 \%)$ \\
Total dislocation of the IOL & Scleral fixation of present IOL, $n=$ \\
Type of correcting surgery & $132(80 \%)$ \\
& Scleral fixation after IOL exchange, \\
& $n=33(20 \%)$
\end{tabular}

$\overline{C T R}$ capsule tension ring, $B C V A$ best corrected visual acuity, $\log M A R$ logarithm of the minimal angle of resolution, $I O P$ intraocular pressure, $I O L$ intraocular lens

${ }^{a}$ Cases with at least one of the following: previous glaucoma surgery, ongoing topical treatment for reducing IOP and IOP of at least $23 \mathrm{mmHg}$

In the majority of subjects, i.e. $80 \%$, a repositioning of the present IOL with ab externo suturing to the scleral wall was used. In this cohort an anterior vitrectomy was performed in 13 cases $(9.8 \%)$ only. Pars plana vitrectomy and IOL exchange with scleral suturing of the replacement posterior chamber IOL was used in the remaining 33 cases including the 25 participants who had a complete IOL dislocation. Generally, the surgery was successful in aligning the optic of the present or the replaced IOL to the pupillary zone and the postoperative course was calm. Reoperations during the follow-up apart from cyclophotocoagulation treatments were very few (Table 3 ). Of note is that re-fixation of IOL was necessary in but six cases and exchange surgery in merely one case. No incidents of endophthalmitis, retinal detachment, significant vitreous haemorrhage or extended periods of iritis were identified, but 13 cases $(8 \%)$ suffered from chronic macular oedema at the 1-year follow-up.

Table 3 presents follow-up data at 1 year. BCVA was statistically significantly improved to $0.32 \pm 0.41 \log$ MAR, $p<0.001$, paired $T$-test, with no difference between IOL reposition or exchange. Expressed in Snellen decimal, the proportion of patients with BCVA 0.5 or better improved to $72.1 \%$ as compared with $35.7 \%$ at presentation. The corresponding proportions for median Snellen worse than 0.1 was $18.1 \%$ at baseline and $6.7 \%$ at the follow-up, preexisting macular pathology being the main explanation of a poor visual result. The only risk factors for a final BCVA worse than $\log$ MAR 0.3 were baseline $\log$ MAR vision worse than 0.3 ( $p=0.001 ; 95 \%$ CI: $1.969,12.694)$ and age 85 years or more $(p=0.003 ; 95 \%$ CI: $1.068,4.862)$.

IOP statistically significantly decreased to $18.4 \pm 6.0$ mmHg at follow-up, $p=0.02$, paired $T$-test. At 1 year, there was no statistically significant difference between the group with a repositioned IOL, $18.7 \pm 5.9 \mathrm{mmHg}$, and the group with IOL exchange, $17.24 \pm 5.86 \mathrm{mmHg}, p=0.2$, un-paired $T$-test. When considering patients with a pressure issue at the follow-up, the proportion was exactly the same, $47.3 \%$, as right before the intervention, with most, i.e. $82 \%$, of patients affected at baseline remaining in this category. IOP in eyes with an IOP issue at presentation, $n=78$, decreased significantly from $24.79 \pm 9.42 \mathrm{mmHg}$ to $18.93 \pm 6.36$ $\mathrm{mmHg}, \quad p<0.001$, paired $T$-test. Conversely, in cases without a pressure issue at presentation, $n=87$, IOP increased markedly from $15.93 \pm 3.18 \mathrm{mmHg}$ to $18.01 \pm$ $5.46 \mathrm{mmHg}, p=0.001$, paired $T$-test. IOP lowering medication was used by $30.3 \%$ of cases at baseline and by $35.7 \%$ at the 1-year visit. Adding to these, sixteen subjects without treatment were found to have an IOP of $23 \mathrm{mmHg}$ or more at the 1-year follow-up and were then started on IOP reducing eye drops. Regarding glaucoma surgery, $7 \%$ were operated with cyclophotocoagulation simultaneously with the dislocation correction and $3.6 \%$ in the year to follow. In a logistic regression analysis, the risk of having an IOP of $23 \mathrm{mmHg}$ or more at the follow-up was independently increased only in patients aged 84 years or less at the corrective procedure, $(p=0.018 ; 95 \% \mathrm{CI}: 1.303,12.826)$. With the same multivariate statistical model, the risk of having a pressure issue at the 1-year follow-up was found to be independently increased if there was a pressure issue at baseline $(p<0.001 ; 95 \% \mathrm{CI}: 8.941,43.908)$ or if the IOL was repositioned ab externo $(p=0.006 ; 95 \%$ CI: 1.606 , 15.913).

\section{Discussion}

This prospective study evaluates the 12-month outcome of surgical repair of late in-the-bag IOL dislocation in 165 eyes, exceeding the numbers of cases that have been included in studies with similar objectives [7-12]. The sample size allowed for multivariate analyses of putative determinants for main outcome measures, an analytical method that has so far been applied only in one published report [7]. In terms of visual results, the mean postoperative logMAR BCVA of 0.32 is on a par with previous studies. The only predisposing factor found for a worse visual 
Table 3 Data at 1-year followup., $n=165$

\section{BCVA}

IOP

Topical treatment for reducing IOP

Pressure issue ${ }^{\mathrm{a}}$

Procedure to lower the IOP at or after surgical correction

Reattachment of the IOL after surgery

IOL exchange

Addressed vitreous problems after surgery
$0.32 \pm \log$ MAR Snellen decimal BCVA 0.5 or better $72.1 \%(72.7 \%$ in the reposition group and $69.7 \%$ in the exchange group)

$18.44 \pm 5.96$

$n=59(35.8 \%)$

$n=78(47.3 \%)$

$n=18(10.9 \%$; all cyclophotocoagulation)

$n=6(3.6 \%)$

$n=1(0.6 \%)$

YAG treatment of strands, $n=6(3.6 \%)$

Anterior vitrectomy, $n=1(0.6 \%)$

Pars plana vitrectomy, $n=1(0.6 \%)$

$B C V A$ best corrected visual acuity, $\log M A R$ logarithm of the minimal angle of resolution, IOP intraocular pressure $I O L$ intraocular lens

${ }^{\mathrm{a} C}$ Cases with at least one of the following: ongoing topical treatment for reducing IOP, IOP of at least 23 $\mathrm{mmHg}$ and previous glaucoma surgery

outcome were baseline BCVA worse than 0.3 logMAR and age 85 years or more, most probably reflecting presence of ocular co-morbidity which was not specifically analysed as a co-variate but found to be frequent among those not attaining $\log$ MAR vision of 1.0 at follow-up. Our study could not prove a statistically significantly different BCVA among patients that had posterior chamber IOL replacement and PPV, the latter comprising a mere $20 \%$ of the series, compared to those that had a straightforward repositioning procedure of the present IOL. Similarly in other studies, no difference in visual results was shown between scleral fixation in situ of the lens or replacement with an anterior chamber IOL $[8,9,12]$ or an iris claw lens placed behind the pupil [10]. In yet another of the cited publications, a posterior approach with pars plana vitrectomy and externalised haptics of the dislocated IOL apparently did not yield differences in visual outcome when compared with an anterior ab externo approach similar to the one we mainly used [7]. In terms of safety, the techniques employed in our study and those described in the referred papers are deemed satisfactory. Occasionally, endophthalmitis and retinal detachment have been reported $[7,8]$, in contrast to the lack of such events found presently.

There is a clear association between late in-the-bag dislocated IOLs on the one hand and the presence of pseudoexfoliations, IOP problems and/or frank glaucoma on the other hand $[8,9,11,12,14]$. One may speculate that the rise in IOP may be caused by the instability of the iris-IOLdiaphragm which could exert physical strain on the trabecular meshwork. When reviewing the aggregated report of the Swedish National Cataract Register from 2002, the calculated "reference" year of the cataract operation for our cohort, glaucoma as a co-morbidity was reported to be $10 \%$ (data on-line through user ID-login access). Our choice of variables did not include glaucoma per se but the parameter "IOP issue" including any one of previous glaucoma surgery, ongoing medical hypotensive treatment or an IOP of $23 \mathrm{mmHg}$ or more almost reached 50\% throughout the course of the study, consistent with the reported glaucoma rate in comparable studies $[8,9,11,12,14]$. Although we detected a general IOP decrease from baseline to follow-up in line with other reports, the role of the correcting surgery itself in this development is not clear. Our observation that more patients were taking pressure reducing medication at the follow-up, $35.7 \%$, than at presentation, $30 \%$, concurs with relevant studies in the field $[9,11,12]$. As mentioned, a raised IOP was subject to various interventions during the course of the study and a clearcut impact of preoperative factors or the surgical technique on a follow-up IOP $\geq 23$ $\mathrm{mmHg}$ was not found save for age of 84 years or less, the significance of which is unclear. We thus suggest that the composite variable "IOP issue" may give additional insights into this complex matter. Risk factors for having an IOP issue at follow-up were the presence of an IOP issue at baseline and using IOL repositioning from the anterior approach as correction technique. The latter indicates that a lens exchange with a PPV may protect from IOP problems. A similar observation was made in the prospective study by Kristianslund et al., where patients with a dislocated IOL within reach via the anterior segment were allocated to either repositioning of the present IOL or lens exchange to an iris claw lens placed behind the iris together with a PPV. In the latter cohort, IOP tended to be more decreased than in the former group [11]. In two retrospective studies, however, lens exchange with PPV was not shown to be associated with a more favourable IOP development but in those 
studies the replacement IOL was inserted in the anterior chamber $[9,12]$. In reviewing our own results, it should be emphasised that 25 of the 33 cases operated with PPV and posterior chamber lens exchange had a total IOL dislocation to the vitreous. In fact, this condition was associated with a lack of an IOP issue both at baseline, $76 \%$, and at the follow-up, $88 \%$, both proportions being statistically significantly higher in uni-variate comparisons with cases with an IOL within the optical axis. Still, the parameter IOL exchange with a PPV and not the parameter total IOL dislocation was shown to protect against an IOP issue at follow-up in the logistic regression model. The impact of total dislocation on subsequent IOP evolvement has not been previously elucidated. Either such an analysis was not presented [7], or the number of cases with this condition was too limited $[8,9,12]$.

In summary, this study demonstrates that the ab externo repositioning manoeuvre is a convenient, effective and safe option for in-the-bag subluxated IOLs that are within reach for an anterior approach. In the time that has passed since study completion, it has remained the method of choice at our institution and recently it was shown to be more affordable than lens replacement with an iris claw implant placed behind the pupil in patients where the subluxation was manageable from a limbal approach [17]. As for totally subluxated IOLs in our study as well as for a few patients whose IOL was operable via the anterior chamber, explantation was followed by implantation with a posterior chamber PMMA IOL anchored to the sclera which proved to be equally successful in terms of visual rehabilitation. This method has since been modified in our clinic since the replacement IOL is now three-piece acrylic, the prolene haptics of which are externalised and either inserted in scleral tunnels or covered by scleral flaps [7, 18]. Anterior chamber implants may seem as a convenient alternative in this situation but our clinical policies are clearly against their use given the long-term risk for endothelial cell compromise [19]. Regarding IOP in the face of an in-thebag subluxated IOL, the other focus of this study, the main conclusion is that it does not appear to be lowered by our favoured $a b$ externo repositioning procedure itself. In contrast, IOL exchange with a PPV was associated with absence of an IOP issue at follow-up. Beyond doubt, the finding that IOL exchange in combination with a PPV may counteract persistent IOP problems and that a total dislocation of the IOL may contribute to this evolvement should spur larger prospective investigations similar to ours. Such projects should preferably involve longer follow-up periods and could benefit from an application of our suggested composite "IOP issue" variable. No matter what evidence such studies may provide, long-term monitoring of IOP with different intervals depending on the severity of the problem seems mandatory for the vast majority of patients having undergone surgical management of in-the-bag subluxation of the IOL.

\section{Summary}

\section{What was known before}

- Correction of visually impairing late-in-the-bag dislocated intraocular lenses, be it with a repositioning procedure or a lens exchange, leads to a considerable vision improvement.

- Intraocular pressure (IOP) is usually lower after the surgical correction than at presentation. It is not certain, however, that the surgical intervention by itself is responsible for the reduction, since medical glaucoma treatment is maintained or initiated and even glaucoma surgery is performed at the operation or during the follow-up.

\section{What this study adds}

- By creating a tentative composite parameter, IOP issue, consisting of at least any one of previous glaucoma surgery, on-going IOP reducing treatment and/or IOP of $23 \mathrm{mmHg}$ or more, the need for continuous IOP monitoring after the surgery may be more precisely defined.

- IOP issue at presentation and IOL repositioning with an anterior approach were predictors of an IOP issue at the 1-year follow-up.

Funding $\mathrm{AD}$ and MP received funding through the regional agreement on Medical Training and Clinical Research (ALF) between the Stockholm County Council and Karolinska Institutet (20120623). AD and MP received funding through the regional agreement on Medical Training and Clinical Research (ALF) between the Stockholm County Council and Karolinska Institutet (20120623).

\section{Compliance with ethical standards}

Conflict of interest The authors declare that they have no conflict of interest.

Publisher's note Springer Nature remains neutral with regard to jurisdictional claims in published maps and institutional affiliations.

\section{References}

1. Mönestam E. Incidence of dislocation of intraocular lenses and pseudophakodonesis 10 years after cataract surgery. Ophthalmology. 2009; 116:2315-20. 
2. Dabrowska-Kloda K, Kloda T, Boudiaf S, Jakobsson G, Stenevi $\mathrm{U}$. Incidence and risk factors of late in-the-bag intracular lens dislocation: evaluation of 140 eyes between 1992 and 2012. J Cataract Refract Surg. 2015;41:1376-82.

3. Behndig A, Montan P, Stenevi U, Kugelberg M, Lundström M. One million cataract surgeries: Swedish National Cataract Register 1992-2009. J Cataract Refract Surg. 2011;37:1539-45.

4. Davis D, Brubaker J, Espander L, Stringham J, Crandall A, Werner L, et al. Late in-the-bag spontaneous intraocular lens dislocation. Evaluation of 86 consecutive cases. Ophthalmology. 2009;116:664-70.

5. Bhattacharjee H, Bhattacharjee J, Das D, Singh M, Sukumar P, Misra DK. Pathology and immunohistochemistry of capsular bag in spontaneously late dislocated capsular bag-intraocular lens complex. Indian J Ophthalmol. 2017;65:949-54.

6. Jakobsson G, Zetterberg M, Lundström M, Stenevi U, Grenmark $\mathrm{R}$, Sundelin K. Late dislocation of in-the-bag and out-of-the bag intraocular lenses: ocular and surgical characteristics and time to lens repositioning. J Cataract Refract Surg. 2010;36:1637-44.

7. Jakobsson G, Zetterberg M, Sundelin K, Stenevi U. Surgical repositioning of intraocular lenses after late dislocation: complications, effect on intraocular pressure, and visual outcome. J Cataract Refract Surg. 2013;39:1879-85.

8. Östern A, Sandvik G, Drolsum L. Late-in-the-bag intraocular lens dislocation in eyes with pseudoexfoliation syndrome. Acta Ophthalmol. 2014;92:184-91.

9. Shingleton BJ, Yang Y, O'Donaghue MW. Management and outcomes of intraocular lens dislocation in patients with pseudoexfoliations. J Cataract Refract Surg. 2013;39:984-93.

10. Kristianslund $\mathrm{O}$, Råen $\mathrm{M}$, Östern AE, Drolsum L. Late-in-the-bag intraocular lens dislocation. A randomized clinical trial comparing lens exchange and lens repositioning. Ophtalmology. 2017;124:151-9.
11. Kristianslund $\mathrm{O}$, Råen M, Östern AE, Drolsum L. Glaucoma and intraocular pressure in patients operated for late-in-thebag intraocular lens dislocation. Am J Ophthalmol. 2017;176: 219-27.

12. Bulnes BL, de Rojas Silva V, Moore RL. Intraocular pressure changes before and after surgery for spontaneous in-the-bag intraocular lens dislocation. J Cataract Refract Surg. 2019;45: 305-11.

13. Fernández-Buenaga R, Aliò JL, Pérez-Ardoy AL, Larosa-Quesada A, Pinilla-Cortés L, Barraquer R, et al. Late in-the-bag intraocular lens dislocation requiring explantation: risk factors and outcomes. Eye. 2013;27:796-801.

14. Leung VC, Singh H, Ahmed IIK. Inter-eye difference in patients with pseudoexfoliation syndrome presenting with intraocular lens dislocation. Ophthalmology. 2015;122:480-85.

15. Chan CC, Crandall AS, Ahmed II. Ab externo scleral loop fixation for posterior chamber intraocular lens decentration: clinical results. J Cataract Refract Surg. 2006;32:121-8.

16. Grimfors M, Lundström M, Höijer J, Kugelberg M. Intraoperative difficulties, complications and self-assessed visual function in cataract surgery. Acta Ophthalmol. 2018;96:592-9.

17. Kristianslund O, Dalby M, Moe M, Drolsum L. Cost-effectiveness analysis in a randomised trial of late-in-the-bag intraocular lens surgery: repositioning versus exchange. Acta Ophthalmol. 2019; e-pub ahead of print Apr9 2019; https://doi.org/10.1038/aos14108

18. Gabor SG, Pavlidis MM. Sutureless intrascleral posterior chamber intraocular lens fixation. J Cataract Refract Surg. 2007;33: 1851-54.

19. Bande MF, Garcia-Garces I, Paniagua L, Ruiz-Oliva F, Pineiro A, Blanco-Teijero MJ. Evaluation of vitrectomy and reimplantation following late dislocation of the intraocular lens-capsule bax complex. Retina; 2017;37:925-29. 